\title{
METAMORPHOSES OF RESONANCE CURVES IN SYSTEMS OF COUPLED OSCILLATORS
}

\author{
JAn KyZIOE, AndRZEJ OKNIŃSKI \\ Kielce University of Technology, Kielce, Poland \\ e-mail:kyziol@tu.kielce.pl; fizao@tu.kielce.pl
}

\begin{abstract}
We study dynamics of two coupled periodically driven oscillators in a general case and compare it with two simplified models. Periodic steady-state solutions to these system equations are determined within the Krylov-Bogoliubov-Mitropolsky approach. Amplitude profiles are computed. These two equations, each describing a surface, define a 3D curve - intersection of these surfaces. In the present paper, we analyse metamorphoses of amplitude profiles induced by changes of control parameters in three dynamical systems studied. It is shown that changes of the dynamics occur in the vicinity of singular points of these 3D curves.
\end{abstract}

Keywords: coupled oscillators, amplitude profiles, singular points

\section{Introduction}

We study dynamics of two coupled nonlinear oscillators, one of which being driven by an external periodic force. Equations of motion are

$$
\begin{aligned}
& m \ddot{x}-V(\dot{x})-R(x)+V_{e}(\dot{y})+R_{e}(y)=F(t) \\
& m_{e}(\ddot{x}+\ddot{y})-V_{e}(\dot{y})-R_{e}(y)=0
\end{aligned}
$$

where $x$ is the position of primary mass $m, y$ is the relative position of another mass $m_{e}$ attached to $m$ and $R, V$ and $R_{e}, V_{e}$ are nonlinear elastic restoring and nonlinear forces of internal friction for masses $m, m_{e}$, respectively (we use convention $\dot{x} \equiv d x / d t$, etc.). A dynamic vibration absorber is a typical mechanical model described by (1.1) (in this case $m$ is usually much larger than $m_{e}$ ) (Den Hartog, 1985; Oueini et al., 1999).

Dynamics of coupled, externally and/or parametrically driven oscillators, is very complex. Indeed, there are many interesting nonlinear phenomena present in this class of dynamical systems. There exists a large body of analytical and numerical studies documenting multistability, symmetry breaking, attractors merging, synchronisation, existence of exotic attractors and various transitions to chaos (Bi, 2004; Brezetskyi et al., 2015; Chen and $\mathrm{Xu}, 2010$; Danzl and Moehlis, 2010; Dudkowski et al., 2014; Kuznetsov et al., 2009; Laxalde et al., 2006; McFarland et al., 2005; Pikovsky et al., 2003; Sabarathinam et al., 2013; Warmiński, 2010).

In our earlier papers, we have designed a method based on the theory of singular points of 2D curves, permitting computation of parameter values at which qualitative changes (metamorphoses) of 2D amplitude curves occur, see Kyzioł and Okniński (2013) and references therein. We have also shown that metamorphoses of amplitude profiles are visible in bifurcation diagrams as qualitative changes of dynamics (bifurcations). Recently, our approach has been generalized to the case of 3D resonance curves and applied to compute bifurcations in dynamical system (1.1) with small nonlinearities in the main mass frame (Kyzioł, 2015). It is thus possible to treat system (1.1) as a small perturbation of model with linear functions $R(x), V(\dot{x})$ analyzed in Kyzioł and Okniński (2013) (let us recall that in this case internal motion can be separated 
off, leading to a simpler equation for the corresponding amplitude profile) and use the results obtained by Kyzioł and Okniński (2013). We show that the method is a powerful tool to predict bifurcations of nonlinear resonances present in such dynamical systems.

In the paper by Awrejcewicz (1995), the author outlined a programme, based on the Implicit Function Theorem, to "define and find different branches intersecting at singular points" of amplitude equations. In the present paper, we are working in a more general context of theory of singular points of algebraic curves (Wall, 2004; Hartmann, 2003).

We investigate the following hierarchy of dynamical systems of form (1.1): we consider functions $R, V, R_{e}, V_{e}$ for which (a) system of equations can be reduced to one second-order effective equation of relative motion, (b) fourth-order equation for variable $y$ can be separated off, (c) it is impossible to separate variables. We analyse approximate analytic solutions (amplitude profiles) obtained within the Krylov-Bogoliubov-Mitropolsky (KBM) method (Nayfeh, 1981; Awrejcewicz and Krysko, 2006), using theory of algebraic curves. More exactly, singular points of amplitude profiles are computed. We demonstrate that qualitative changes of dynamics, referred to as metamorphoses, induced by changes of control parameters, occur in neighbourhoods of singular points of amplitude profiles, see also Kyzioł and Okniński $(2011,2013)$ and references therein.

The paper is organized as follows. In the next Section, equations (1.1), (2.1) are transformed into non-dimensional form. In Section 4, implicit equations for resonance surfaces $A(\omega), B(\omega)$ are derived within the Krylov-Bogoliubov-Mitropolsky approach, where the amplitudes $A, B$ correspond to small and large masses, respectively. The problem is more difficult than before because these two equations are coupled. In Section 5, we review necessary facts from theory of algebraic curves which are used to compute singular points on three-dimensional resonance curve (intersection of resonance surfaces $A(\omega), B(\omega)$ ). In Section 6, computational results are presented. Our results are summarized in the last Section.

\section{Equations of motion}

In what follows the function $F(t)$ is assumed in form $F(t)=f \cos (\omega t)$. When all the functions $R, V, R_{e}, V_{e}$ are nonlinear, namely

$$
\begin{array}{ll}
R(x)=-\alpha x-\gamma x^{3} & R_{e}(y)=-\alpha_{e} y-\gamma_{e} y^{3} \\
V(\dot{x})=-\nu \dot{x}-\beta \dot{x}^{3} & V_{e}(\dot{y})=-\nu_{e} \dot{y}-\beta_{e} \dot{y}^{3}
\end{array}
$$

then we deal with the general case of Eq. (1.1). For linear functions $R, V$

$$
\begin{array}{ll}
R(x)=-\alpha x & R_{e}(y)=-\alpha_{e} y-\gamma_{e} y^{3} \\
V(\dot{x})=-\nu \dot{x} & V_{e}(\dot{y})=-\nu_{e} \dot{y}+\beta_{e} \dot{y}^{3}
\end{array}
$$

it is possible to separate off the variable $y$ to obtain the following equation for relative motion (Kyzioł and Okniński, 2013)

$$
\widehat{L}\left(\mu \ddot{y}-V_{e}(\dot{y})-R_{e}(y)\right)+\epsilon m_{e} \widehat{K} y=F \cos (\omega t)
$$

where

$$
\begin{array}{lll}
\widehat{L}=M \frac{d^{2}}{d t^{2}}+\nu \frac{d}{d t}+\alpha & \widehat{K}=\left(\nu \frac{d}{d t}+\alpha\right) \frac{d^{2}}{d t^{2}} & F=m_{e} \omega^{2} f \\
\epsilon=\frac{m_{e}}{M} & \mu=\frac{m m_{e}}{M} & M=m+m_{e}
\end{array}
$$

Finally, assuming $m_{e} \ll m$, i.e. $\epsilon \ll 1$, we can reject the term proportional to $\epsilon$ to obtain an approximate equation which can be integrated partly to yield the effective equation (Kyzioł and Okniński, 2011) 


$$
\begin{aligned}
& \mu \ddot{y}+\nu_{e} \dot{y}-\beta_{e} \dot{y}^{3}+\alpha_{e} y+\gamma_{e} y^{3}=F(t) \\
& F(t)=\frac{-m_{e} \omega^{2} f}{\sqrt{M^{2}\left(\omega^{2}-\frac{\alpha}{M}\right)^{2}+\nu^{2} \omega^{2}}} \cos (\omega t+\delta)
\end{aligned}
$$

where transient states are neglected.

\section{Equations in non-dimensional form}

Equations (1.1), (2.1) are transformed into non-dimensional form (Kyzioł, 2015). We introduce non-dimensional time $\tau$ and frequency $\Omega$ and rescale variables $x, y$

$$
t=\sqrt{\frac{\mu}{\alpha_{e}}} \tau \quad \omega=\sqrt{\frac{\alpha_{e}}{\mu}} \Omega \quad x=\sqrt{\frac{\alpha_{e}}{\gamma_{e}}} u \quad y=\sqrt{\frac{\alpha_{e}}{\gamma_{e}}} z
$$

to get

$$
\begin{aligned}
& \ddot{u}+\widehat{H} \dot{u}+c \dot{u}^{3}+\widehat{a} u+d u^{3}-\widehat{\kappa}\left(h \dot{z}+b \dot{z}^{3}+z+z^{3}\right)=\lambda \cos (\Omega \tau) \\
& \ddot{z}+h \dot{z}+b \dot{z}^{3}+z+z^{3}-\widehat{H} \dot{u}-c \dot{u}^{3}-\widehat{a} u-d u^{3}=-\lambda \cos (\Omega \tau)
\end{aligned}
$$

and new parameters read

$$
\begin{array}{llll}
a=\frac{\mu \alpha}{M \alpha_{e}} & b=\frac{\beta_{e}}{\gamma_{e}}\left(\frac{\alpha_{e}}{\mu}\right)^{\frac{3}{2}} & c=\frac{\beta\left(\alpha_{e}\right)^{\frac{3}{2}}}{\sqrt{\mu} m \gamma_{e}} & d=\frac{\mu \gamma}{m \gamma_{e}} \\
h=\frac{\nu_{e}}{\sqrt{\mu \alpha_{e}}} & H=\frac{\nu}{M} \sqrt{\frac{\mu}{\alpha_{e}}} & G=\frac{1}{\alpha_{e}} \sqrt{\frac{\gamma_{e}}{\alpha_{e}}} f & \kappa=\frac{m_{e}}{m} \\
\lambda=\frac{\kappa}{\kappa+1} G & \widehat{H}=H(1+\kappa) & \widehat{a}=a(1+\kappa) & \widehat{\kappa}=\frac{\kappa}{\kappa+1}
\end{array}
$$

where

$$
M=m+m_{e} \quad \mu=\frac{m m_{e}}{M} \quad \dot{u} \equiv \frac{d u}{d \tau} \quad \dot{z} \equiv \frac{d z}{d \tau}
$$

Note that $\ddot{u}$ is eliminated from the second of Eqs. (1.1).

\section{Nonlinear resonances}

System of equations (3.2) is written in form

$$
\begin{aligned}
& \frac{d^{2} u}{d \tau^{2}}+\Omega^{2} u+\varepsilon(\sigma u+g(\dot{u}, u, \dot{z}, z, \tau))=0 \\
& \frac{d^{2} z}{d \tau^{2}}+\Omega^{2} z+\varepsilon(\sigma z+k(\dot{u}, u, \dot{z}, z, \tau))=0
\end{aligned}
$$

where

$$
\begin{array}{lllll}
\varepsilon \sigma=\Theta^{2}-\Omega^{2} & \widehat{H}=\varepsilon \widehat{H}_{0} & \widehat{a}=\varepsilon \widehat{a}_{0} & b=\varepsilon b_{0} & c=\varepsilon c_{0} \\
\Theta^{2}=\varepsilon \Theta_{0}^{2} & d=\varepsilon d_{0} & h=\varepsilon h_{0} & \varepsilon \delta_{0}=1 & \lambda=\varepsilon \lambda_{0}
\end{array}
$$

and functions $g(\dot{u}, u, \dot{z}, z, \tau), k(\dot{u}, u, \dot{z}, z, \tau)$ are defined in (Kyzioł, 2015). Equations (4.1) have been prepared in such a way that for $\varepsilon=0$ the solutions are $u(\tau)=B \cos (\Omega \tau+\psi)$, $z(\tau)=A \cos (\Omega \tau+\varphi)$. 
We shall now look for 1: 1 resonance using the Krylov-Bogoliubov-Mitropolsky (KBM) perturbation approach (Nayfeh, 1981; Awrejcewicz and Krysko, 2006). For a small nonzero $\varepsilon$, the solutions to Eqs. (4.1) are assumed in form

$$
\begin{aligned}
& u(\tau)=B \cos (\Omega \tau+\psi)+\varepsilon u_{1}(B, \psi, \tau)+\ldots \\
& z(\tau)=A \cos (\Omega \tau+\varphi)+\varepsilon z_{1}(A, \varphi, \tau)+\ldots
\end{aligned}
$$

with slowly varying amplitudes and phases

$$
\begin{aligned}
\frac{d A}{d \tau} & =\varepsilon M_{1}(A, \varphi)+\ldots & \frac{d B}{d \tau} & =\varepsilon P_{1}(B, \psi)+\ldots \\
\frac{d \varphi}{d \tau} & =\varepsilon N_{1}(A, \varphi)+\ldots & \frac{d \psi}{d \tau} & =\varepsilon Q_{1}(B, \psi)+\ldots
\end{aligned}
$$

Proceeding as described in (Kyzioł, 2015), we obtain finally equations for the amplitudes $A, B$

$$
L_{1}(A, B, \Omega ; \Lambda)=0 \quad L_{2}(A, B, \Omega ; \Lambda)=0
$$

where $\Lambda$ denotes parameters and

$$
\begin{aligned}
& L_{1}=Z\left(\left(\eta_{4}^{2}+u_{2}^{2}\right)\left(u_{1}^{2}+\eta_{2}^{2}\right)+\widehat{\kappa} X^{2} u_{3}\right)-(\widehat{\kappa}-1)^{2}\left(u_{1}^{2}+\eta_{2}^{2}\right) \lambda^{2} \\
& u_{1}=\widehat{\kappa} X+\eta_{1} \quad u_{2}=\widehat{\kappa} X+\eta_{3} \quad u_{3}=\widehat{\kappa} X^{2}+2 \eta_{2} \eta_{4}-2 u_{1} u_{2} \\
& L_{2}=\frac{Y}{(\widehat{\kappa}-1)^{2}}\left[u_{4}^{2}+\left(\widehat{\kappa} X u_{5}+\eta_{1} \eta_{3}-\eta_{2} \eta_{4}\right)^{2}\right]-\lambda^{2} X^{2} \\
& u_{4}=\widehat{\kappa} X\left(\eta_{2}+\eta_{4}\right)+\eta_{1} \eta_{4}+\eta_{2} \eta_{3} \quad u_{5}=(\widehat{\kappa}-1) X+\eta_{1}+\eta_{3} \\
& \eta_{1}=\left(\frac{3}{4} Y+1-X\right)(\widehat{\kappa}-1) \quad \eta_{2}=\Omega\left(\frac{3}{4} b X Y+h\right)(\widehat{\kappa}-1) \\
& \eta_{3}=\left(\widehat{a}+\frac{3}{4} d Z-X\right)(\widehat{\kappa}-1) \quad \eta_{4}=\Omega\left(\frac{3}{4} c X Z+\widehat{H}\right)(\widehat{\kappa}-1) \\
& X=\Omega^{2} \quad Y=A^{2} \quad Z=B^{2}
\end{aligned}
$$

If we put $c=d=0$ in Eqs.(4.6), (4.7) and (4.8) (or $\beta=\gamma=0$ in Eqs. (2.1)) then the function $L_{2}$ becomes independent on $B$. In this case, it is possible to separate variables in Eqs. (1.1), (2.2) obtaining the fourth-order effective equation for the small mass (Kyzioł and Okniński, 2013). The function $L_{2}$, defined above, for $c=d=0$ is equal to the function $L(X, Y)$ defined in Eq. (4.1) in (Kyzioł and Okniński, 2013).

\section{Metamorphoses of the amplitude profiles}

In the preceding Section, we have obtained two implicit equations (4.5) for amplitude profiles. Each of these equations describes a surface in a three dimensional space $(A, B, \Omega)$. Intersection of the surfaces $L_{1}=0, L_{2}=0$ is a $3 \mathrm{D}$ curve, and in singular points of this curve all three minors of the rectangular matrix

$$
\mathbf{M}=\left[\begin{array}{lll}
L_{1, A}^{\prime} & L_{1, B}^{\prime} & L_{1, \Omega}^{\prime} \\
L_{2, A}^{\prime} & L_{2, B}^{\prime} & L_{2, \Omega}^{\prime}
\end{array}\right]
$$

are zero (Hartmann, 2003), where $L_{1, A}^{\prime}=\partial L_{1} / \partial A$, etc. Equations (4.5) and these conditions are used to compute singular points. We have shown in our previous papers that qualitative changes of dynamics, induced by changes of control parameters, occur in neighbourhoods of singular points of amplitude profiles (Kyzioł and Okniński, 2011, 2013; Kyzioł, 2015). 


\section{Amplitude profiles and bifurcation diagrams}

Applying the KBM method to effective equation (2.4) we obtain approximate formula $y(t)=A \cos (\omega t+\varphi)$ where dependence of $A$ on $\omega$ is given by an implicit equation $F_{1}(A, \omega ; \Lambda)=0$. The form of the function $F_{1}$ can be found in Kyzioł and Okniński (2011). In Fig. 1a, this implicit function is shown just after an isolated point $(A, \omega)=(1.124,1.784)$ has been born.
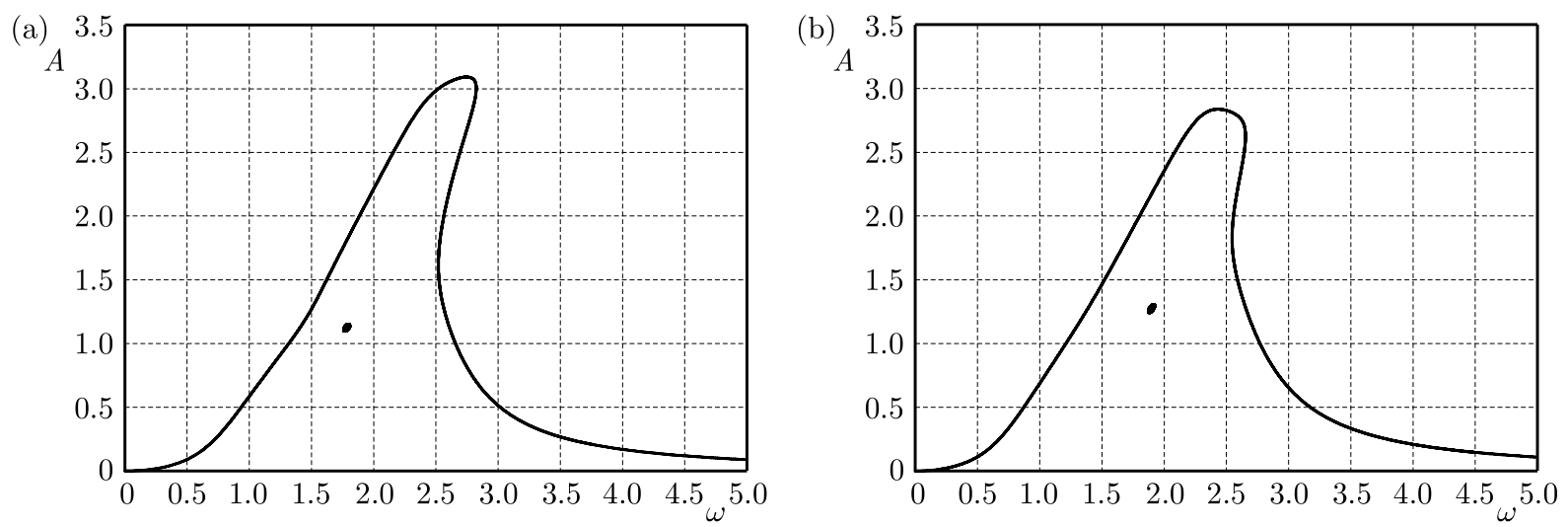

Fig. 1. Amplitude profile $F_{1}(A, \omega ; \Lambda)=0$ (a) and $F_{2}(A, \omega ; \Lambda)=0$ (b) with an isolated point

Then, applying the KBM method to fourth-order equation (2.3) we obtain the corresponding implicit amplitude equation $F_{2}(A, \omega ; \Lambda)=0$. The form of $F_{2}$ has been described in Kyziol and Okniński (2013). In Fig. 1b, we see that an isolated point $(A, \omega)=(1.274,1.899)$ has been just born. Similarity of the amplitude profiles shows that effective equation (2.4) is a good approximation to fourth-order equation (2.3). Bifurcation diagrams show indeed the birth of new branches of solutions in both models (Kyzioł and Okniński, 2011, 2013).

Now we consider the general case with small nonlinearities in the main mass frame, $c=0.001$, $d=0.02$, so that the system of equations (1.1), (2.1) is a small perturbation of model (1.1), (2.2), with other parameters being equal $a=6, b=0.001, h=0.5, H=0.7, \kappa=0.05$, $\gamma=2.011615 \stackrel{d f}{=} \gamma_{c r}$.
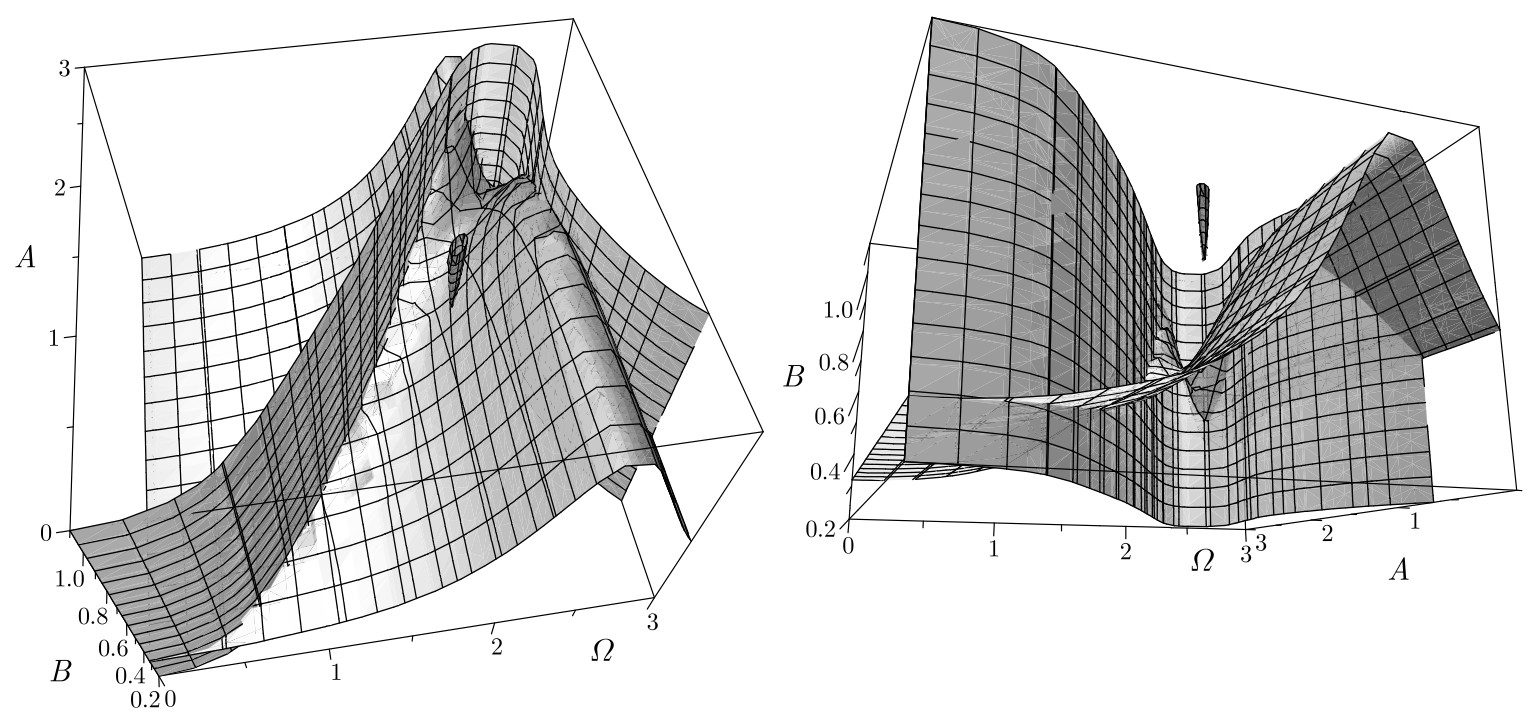

Fig. 2. Resonance surfaces before the singular point is formed, left figure. The conical structure does not intersect the lower surface, right figure

Resonance surfaces (4.5) are shown in Figs. 2 before the singular point is formed, the singular point being $(A, B, \Omega)=(1.276,0.620,1.902)$ and $\gamma=2.015>\gamma_{c r}$, where the surfaces in the right 
figure have been rotated to show that the additional conical surface does not pierce the other surface yet, and, after formation of the singular point, $\gamma=1.995<\gamma_{c r}$, in Fig. 3.

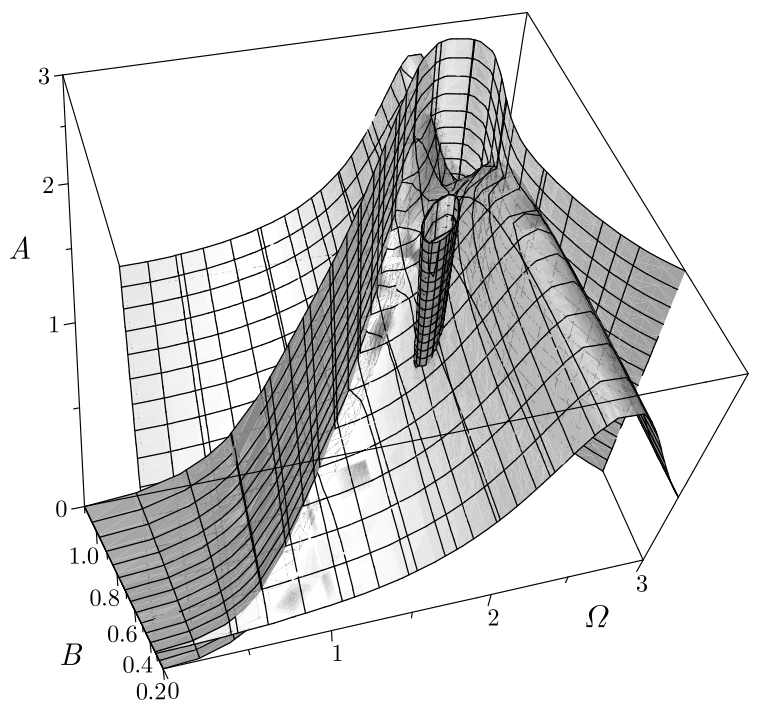

Fig. 3. Resonance surfaces $L_{1}(A, B, \Omega ; \Lambda)=0$ and $L_{2}(A, B, \Omega ; \Lambda)=0$ with an additional tubular structure intersecting the lower surface

The corresponding bifurcation diagrams, one with a new branch near $\Omega=1.9$, are shown in Figs. 4.
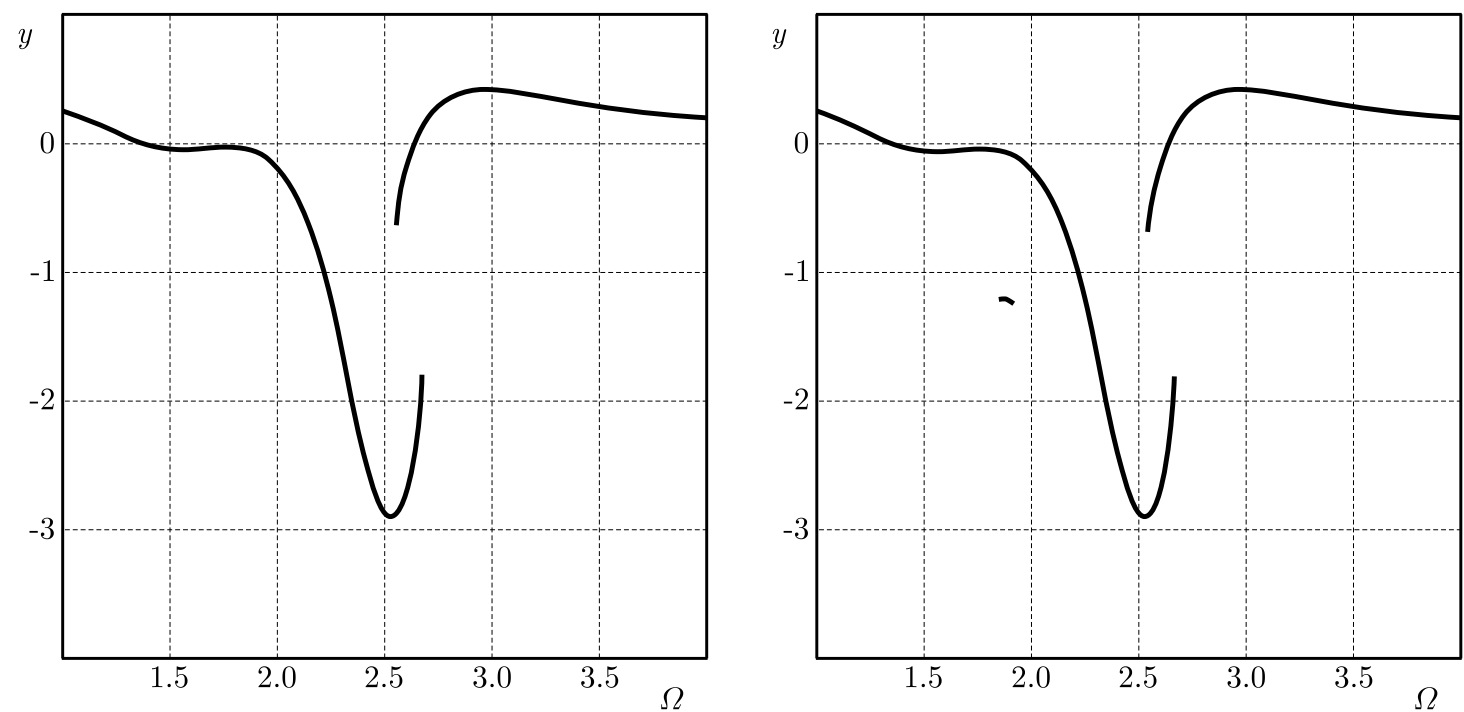

Fig. 4. Bifurcation diagram before formation of the singular point (left figure) and after (right figure)

\section{Discussion}

In the present work, we continue study of the general case of dynamics of two coupled periodically driven oscillators, cf. Eq. (1.1), initiated in Kyzioł (2015). More exactly, we have investigated: (a) model (1.1), (2.1) with small parameters $\alpha, \gamma$; (b) model (1.1), (2.2) with $\alpha=0, \gamma=0$ (in this case, dynamics of small mass can be separated off, see Eq. (2.3)); (c) and approximate effective equation (2.4). 
We have studied the amplitude (resonance) equations for steady states, obtained via the KBM approach, within the theory of singular points of 2D and 3D algebraic curves (Wall, 2004; Hartmann, 2003). Analysis of the resonance curves in cases (b), (c) has been relatively simple since we have been dealing with one implicit equation of form $F(A, \Omega ; \Lambda)=0$, describing a $2 \mathrm{D}$ curve only (Kyzioł and Okniński, 2011, 2013). The general case is more difficult since there are two amplitude equations, $L_{1}(A, B, \Omega ; \Lambda)=0, L_{2}(A, B, \Omega ; \Lambda)=0$, which describe two surfaces. Conditions for singular points of the 3D curve - intersection of these surfaces - are also more complex: they are given by two equations (4.5) and three equations $\operatorname{det}(\mathbf{M})=0$ with matrix $\mathbf{M}$ given by (5.1).

Bifurcations diagrams shown in Figs. 4 confirm that a qualitative change of dynamics - birth of a new branch of a nonlinear resonance - occurs in the neighbourhood of the singular point of the 3D curve defined by amplitude equations (4.5).

\section{Acknowledgement}

The present paper is an extended version of the article published in the Proceedings of the conference: PCM-CMM-2015 - 3rd Polish Congress of Mechanics and 21st Computer Methods in Mechanics, September 8th-11th 2015, Gdańsk, Poland.

\section{References}

1. Awrejcewicz J., 1995, Modified Poincaré method and implicit function theory, [In:] Nonlinear Dynamics: New Theoretical and Applied Results, Awrejcewicz J. (Edit.), Akademie Verlag, Berlin, $215-229$

2. Awrejcewicz J., Krysko V.A., 2006, Introduction to Asymptotic Methods, Chapman and Hall (CRC Press), New York

3. BI Q., 2004, Dynamical analysis of two coupled parametrically excited van der Pol oscillators, International Journal of Non-Linear Mechanics, 39, 33-54

4. Brezetsky S., Dudkowski D., Kapitaniak T., 2015, Rare and hidden attractors in Van der Pol-Duffing oscillators, European Physical Journal Special Topics, 224, 1459-1467

5. Chen H., Xu Q., 2010, Global bifurcations in externally excited autoparametric systems, International Journal of Non-Linear Mechanics, 45, 766-792

6. Danzl P., Moenlis J., 2010, Weakly coupled parametrically forced oscillator networks: existence, stability, and symmetry of solutions, Nonlinear Dynamics, 59, 661-680

7. Den Hartog J.P., 1985, Mechanical Vibrations, 4th ed., Dover Publications, New York

8. Dudkowski D., Maistrenko Y., Kapitaniak T., 2014, Different types of chimera states: An interplay between spatial and dynamical chaos, Physical Review E, 90, 032920

9. Hartmann E., 2003, Geometry and Algorithms for Computer Aided Design, Darmstadt University of Technology, Darmstadt

10. Kuznetsov A.P., Stankevich N.V., Turukina L.V., 2009, Coupled van der Pol-Duffing oscillators: phase dynamics and structure of synchronization tongues, Physica D, 238, 1203-1215

11. Kyzioe J., 2015, Metamorphoses of resonance curves for two coupled oscillators: The case of small non-linearities in the main mass frame, International Journal of Non-Linear Mechanics, $\mathbf{7 6}$, $164-168$

12. KYZıoŁ J., OkniŃski A., 2011, Coupled nonlinear oscillators: metamorphoses of resonance curves. The case of the approximate effective equation, Acta Physica Polonica B, 42, 2063-2076

13. KyzioŁ J., Okniśski A., 2013, Exact nonlinear fourth-order equation for two coupled oscillators: metamorphoses of resonance curves, Acta Physica Polonica B, 44, 35-47 
14. Laxalde D., Thouverez F., Sinou J.-J., 2006, Dynamics of a linear oscillator connected to a small strongly non-linear hysteretic absorber, International Journal of Non-Linear Mechanics, 41, 969-978

15. McFarland D.M., Bergman L.A., Vakakis A.F., 2005, Experimental study of non-linear energy pumping occurring at a single fast frequency, International Journal of Non-Linear Mechanics, 40, 891-899

16. NAYfeH A.H., 1981, Introduction to Perturbation Techniques, John Wiley \& Sons, New York

17. Oueini S.S., Nayfeh A.H., Pratt J.R., 1999, A review of development and implementation of an active nonlinear vibration absorber, Archive of Applied Mechanics, 69, 585-620

18. Pikovsky A., Rosenblum M., Kurths J., 2003, Synchronization: A Universal Concept in Nonlinear Sciences, Cambridge University Press, Cambridge

19. Sabarathinam S., Thamilmaran K., Borkowski L., Perlikowski P., Brzeski P., Stefanski A., Kapitaniak T., 2013, Transient chaos in two coupled, dissipatively perturbed Hamiltonian Duffing oscillators, Communications in Nonlinear Science and Numerical Simulation, 18, 3098-3107

20. Wall C.T.C., 2004, Singular Points of Plane Curves, Cambridge University Press, New York

21. WARmiński J., 2010, Nonlinear normal modes of a self-excited system driven by parametric and external excitations, Nonlinear Dynamics, 61, 677-689 